\title{
Comparison of Endoscopic Injection of Botulinum Toxin and Steriods Immediately after Endoscopic Submucosal Dissection to Prevent Esophageal Stricture: A Prospective Cohort Study
}

Xiaoying Zhou ( $\nabla$ zhouxiaoying0926@njmu.edu.cn ) the first affiliated hospital of nanjing medical university

Han Chen

the first affiliated hospital of Nanjing Medical University

Meihong Chen

The First Affiliated Hospital of Nanjing Medical University

Chao Ding

the first affiliated hospital of Nanjing Medical University

Guoxin Zhang

the first affiliated hospital of nanjing medical university

Xinmin Si

the affiliated hospital of nanjing medical university

\section{Research}

Keywords: endoscopic submucosal dissection, esophageal stricture, endoscopic injection, botulinum toxin type $A$, triamcinolone acetonide, prospective cohort study

Posted Date: February 12th, 2021

DOl: https://doi.org/10.21203/rs.3.rs-206425/v1

License: (a) (i) This work is licensed under a Creative Commons Attribution 4.0 International License. Read Full License 


\section{Abstract}

Background: Widespread endoscopic submucosal dissection (ESD) in early esophageal cancer patients is closely associated with esophageal stricture, which dramatically reduces patients' quality of life and increases huge medical burdens. Endoscopic injection of steroid was proved as a protective method for post-ESD strictures. Other materials such as botulinum toxin type A (BTX-A) may be potential candidates. We conducted this prospective cohort study to compare the efficacy and feasibility of endoscopic injection of BTX-A and triamcinolone acetonide (TA) for the prevention of esophageal stricture.

Methods: Seventy-eight patients with esophageal mucosal defects of more than two thirds of the circumference were successively enrolled and divided into 3 groups: BTX-A group (group A, n=26), TA group (group $B, n=16$ ) and control group (group $C, n=36$ ). Patients in group $A$ were immediately injected with BTX-A after ESD, in group $B$ were immediately injected with TA and in group $C$ received ESD only. Endoscopy was performed when patients reported dysphagia symptoms and at 6 and 12 weeks post-ESD in patients without symptoms. Patients who experienced post-ESD esophageal strictures in all groups received bougie dilation. All patients were followed up for one year.

Results: The proportion of patients developing stricture in BTX-A group was $30.00 \%$ (intention to treat analysis, 9/30) and $26.92 \%$ (per protocol analysis, $7 / 26$ ), in TA group was $40.90 \%$ (intention to treat analysis, $9 / 22$ ) and $43.75 \%$ (per protocol analysis, $7 / 16$ ), and in control group was $84.21 \%$ (intention to treat analysis, $32 / 38)$ and $83.33 \%$ (per protocol analysis, 30/36) $(\mathrm{p}<0.001)$. When further comparing between each of the two groups, the incidence of esophageal stricture was lower in BTX-A group than that in control group $(p<0.001)$, and lower in TA group than that in control group $(p=0.004)$. Furthermore, in entire circumference mucosal defect subgroup, the esophageal stricture was significantly lower in BTXA group than that in TA group (33.3\% vs $100 \%, p=0.0454)$.

Conclusions: Endoscopic injection of BTX-A and TA were effective in preventing post-ESD esophageal strictures and BTX-A injection was particularly effective in entire circumference mucosal defect patients. Multi-centered, randomized prospective study with larger sample size should be conducted. (Clinical trial registration number: ChiCTR2100042970, registered 1 February 2021, retrospectively registered, http://www.chictr.org.cn/listbycreater.aspx)

\section{Background}

Endoscopic resection is globally accepted as a minimally invasive treatment for early esophageal cancer [1]. As an alternative to esophagectomy, endoscopic mucosal resection (EMR) had been originally applied in the treatment for esophageal adenocarcinoma or localized neoplasm [2]. Recently, EMR has been gradually replaced by endoscopic submucosal dissection (ESD), as ESD allows the entire resection of the lesion regardless of its size and has a lower recurrence rate compared to EMR [3]. However, the residual mucosal defect after ESD may cause acute inflammation, deep ulcers, local submucosal fibrous 
connective tissue proliferation, collagen deposition, esophageal wall fibrosis, and esophageal stricture formation $[4,5]$.

The incidence of esophageal strictures after endoscopic resection resulting in large near-circumferential or circumferential esophageal mucosal defects has been reported to be $88-100 \%$ [6]. Although endoscopic balloon dilation is effective for the treatment of strictures, it is performed repeatedly until dysphagia resolves [7]. Repeated dilation not only increases the risk of perforation and bleeding and the burden of society but also reduces the patient's quality of life [8]. A review report investigating 73 studies suggested that oral triamcinolone acetonide (TA) administration, not prophylactic endoscopic balloon dilation alone, was effective in preventing esophagostenosis and reducing the number of repeated endoscopic balloon dilations even after extensive endoscopic resection [9]. Local steroid injection is useful and economy for preventing esophageal stricture, even though it may raise the risk of perforation during dilations [10]. Jing et al concluded for the first time that a single injection of botulinum toxin type A (BTX-A) reduced esophageal stricture rate and the times of bougie dilation procedures, as BTX-A was reported to be used to decrease the fibrosis of a surgical wound and prevent widening of facial scars [11]. BTX-A can also down-regulate the expression of both transforming growth factor-b1 mRNA and transforming growth factor-b1 protein in the esophageal scar tissues, leading to less deposition of both type I and type III collagen in the tissues [12].

A preliminary aim of this study was to determine the relationship between the extent of the esophageal mucosal defect after ESD and the risk of stricture formation. Specifically, we determined the risk of strictures in ESD patients with mucosal defects more than one half of the circumference of the esophagus after ESD treatment. Our prospective study's primary aim was to investigate the efficacy and feasibility of the endoscopic injection of BTX-A and TA for the prevention of esophageal strictures after ESD for early esophageal cancer.

\section{Methods}

\section{Study patients}

Originally, 80 patients with early esophageal cancer who underwent ESD at First Affiliated Hospital of Nanjing Medical University from March 2018 to May 2019 were randomly divided into BTX-A group (group A), TA group (group B) and control group (group C). The inclusion criteria for the study were as follows: 1) preoperative pathology indicating precancerous lesions or carcinoma in situ, 2) mucosal defects exceeding two thirds of the circumference of the esophagus, 3 ) the absence of lymph node metastases confirmed by CT, 4) no contraindications to general intravenous anesthesia, 5) no serious cardiopulmonary disease, 6 ) the patient's signed informed consent. However, we excluded patients who was diagnosed invasive esophageal carcinoma or tumor recurrence. Patients suffering from other diseases of esophagus such as congenital anatomical structure abnormality, esophageal varices, functional esophageal disease, severe reflux esophagitis were also excluded from the study. 
Our study has been approved by the Ethics Committee of First Affiliated Hospital of Nanjing Medical University and written informed consent was obtained from each patient. The ethical approval number for this study was 2018-SR-199. (Clinical trial registration number: ChiCTR2100042970)

\section{Study design}

We designed a prospective cohort study. Before ESD procedure, all patients were prospectively divided into three groups. Patients in group A (BTX-A group, $n=30$ ) received endoscopic BTX-A injection immediately after ESD procedure, patients in group $B(T A$ group, $n=20$ ) received TX injection immediately after $E S D$, and patients in group $C$ (control group, $n=40$ ) received ESD only without subsequent injection. The mucosal defect after ESD was classified into three groups based on the extent of the areas affected: two thirds to three fourths of the esophageal circumference, three fourths to the full esophageal circumference and full circumferential mucosal defect. In either group A, B or C, all patients with full circumferential mucosal defects received oral prednisolone after ESD.

\section{ESD procedures}

Endoscopic procedures were carried out with an upper endoscope with an outer diameter of $9.9 \mathrm{~mm}$ (GIFQ260J; Olympus Medical System Co., Tokyo, Japan). The electrosurgical unit and knife for ESD consisted of a high frequency generator (VIO300; ERBE Elektromedizin, Tubingen, Germany), the HookKnife and the DualKnife (Olympus Co.). Submucosal dissection was carried out with the autocut mode (60 W, effect, 5)

to decrease the burning effect on the resected surface, which could reportedly cause severe stenosis after extensive esophageal ESD. The coagulation mode was used only to stop bleeding and for preventive vascular coagulation.

\section{Endoscopic BTX-A and TA Injection procedure}

Just after dissection and hemostasis, a single-session endoscopic BTX-A or TA injection was administered. BTX-A solution (Lanzhou Institute of Biological Products, Lanzhou, China) was injected in 5 - $\mathrm{mL}$ increments into 10 separate points at the level of the muscularis propria equally spaced along the circumference of the defect with a 25-gauge, 4-mm needle (TOP Corporation, Tokyo, Japan). TA was diluted with $0.9 \% \mathrm{NaCl}$ to a final concentration of $4 \mathrm{mg} / \mathrm{ml}$. A total of $40 \mathrm{mg}(10 \mathrm{ml})$ TA was injected into the deep submucosa of the ulcer base at 10 sites, with a $1 \mathrm{~mL}$ dose at each site. A total of 100 units of BTX-A was diluted with $5 \mathrm{~mL}$ of saline solution (20 units $/ \mathrm{mL}$ ). Where BTX-A or TA, the injections were placed along the junction of the defect and the normal tissue. However, patients with full circumferential mucosal defects was injected superficially into the base of the cautery ulcer. All patients received the same dose of BTX-A (100 units) and TA (40mg), regardless of the lesion size.

\section{Postoperative management and follow-up}

All patients were requested fasting the first day after ESD, and liquid diets for the next several days. Proton pump inhibitor, antibiotic, and hemostatic were routinely used to promote rehabilitation. The occurrence of perforation, bleeding, fever, chest pain, allergy and other adverse events are paid closely 
attention. Patients with entire circumferential ESD were administered a systemic steroid. Oral prednisolone was started at a dose of $30 \mathrm{mg} /$ day on the second day post-ESD, tapered gradually $(30,30$, $30,30,25,25,20,20,17.5,17.5,15,15,12.5,10,7.5$ and $5 \mathrm{mg}$ for 7 days each) and then discontinued 16 weeks (112 days) later. Follow-up endoscopy was scheduled at 6 and 12 weeks after ESD, and telephone follow-up was conducted every week post-ESD for dysphagia and Quality of Life Questionnaire (EORTC QLQ-OES18) scores. Dysphagia was evaluated using the Mellow-Pinkas score as follows: $0=$ no dysphagia, 1 = dysphagia to normal solids, $2=$ dysphagia to soft solids, $3=$ dysphagia to solids and liquids, and 4 = complete dysphagia, even to saliva. Bougie dilation using Savary-Gilliard dilators (WilsonCook Medical, Winston-Salem, NC) was applied as needed whenever the patients complained of dysphagia. In cases of persistent dysphagia, bougie dilation was performed until dysphagia resolved.

\section{Endpoints}

Esophageal stricture is defined as a symptomatic dysphagia and/or impossible passage of a standard endoscope at the stricture. Through endoscopy and telephone follow-up, we recorded the number of patients with stricture in each group, and the number of required dilatation procedures for treatment of a stricture, the dysphagia grading score, the Quality of Life Questionnaire (EORTC QLQ-OES18) score, the asymptomatic remission period, the diameter of a narrow esophagus, and the time of first stenosis occurred. During the asymptomatic remission period, patients had a dysphagia score larger than 2 , and did not need endoscopic dilatation. The primary endpoint of our study was the stricture rate after ESD with or without BTX-A/TA injection. Secondary endpoints were the number of dilatation procedures, the dysphagia and Quality of Life Questionnaire (EORTC QLQ-OES18) scores, the asymptomatic remission period, the diameter of a narrow esophagus, and the time of first stenosis occurred. Since this study was a prospective cohort study, we defined TA and BTX-A injection as different exposure factors, and the endpoints we mentioned above were the indicators we compared.

\section{Statistical analysis}

PASS 15 (Version: 15.0.13) was used for determining sample size in this study. Section of Proportions in PASS for Chi-Square (Contingency Table/Crosstabs) Tests was applied for sample calculation. Based on the $3 * 2$ Contingency Table, Degrees of Freedom was 2 with a power of 0.90 , Alpha of 0.05 , and W (Effect Size) of 0.432049 (calculated by typing an expected stricture rate of $20 \%$ in BTX, $40 \%$ in TA and $80 \%$ in control groups, respectively). We calculated a sample size of at least 68 patients required for statistical analysis. Based on this, we finally enrolled 90 patients when considering an approximately $20 \%$ drop-up rate.

Normality tests were applied by Shapiro-Wilk and Kolmogorov-Smirnov test. To compare the means from three groups, One-Way ANOVA was applied in continuous variables with normal distribution and KruskalWall H-test for those with the abnormal distribution. Chi-Square Test or Fisher's Exact Test were used for in dichotomous variables ( $3 \times 2$ Contingency Tables) to compare treatment effects among three groups. Pairwise comparisons were conducted in Contingency Tables using an adjusted a level after Bonferroni correction method. Logistic regression was performed to identify any significant risk factors of 
esophageal stricture. Statistical analysis was performed with IBM SPSS Statistics for Windows, Version 23.0 (SPSS, Chicago, IL). P values $<0.05$ were considered statistically significant in general comparisons among three groups. For pairwise comparisons, $P$ values $<0.0167(0.05 / 3)$ were considered statistically significant after Bonferroni correction test.

\section{Results}

\section{Patient characteristics}

Ninety patients who fulfilled the inclusion and exclusion criteria with an esophageal defect greater than two thirds of the circumference were enrolled in this study, of whom 30 received BTX-A injection immediately after ESD procedure, 20 received TA injection immediately after ESD procedure and 40 only received ESD. Seven patients were excluded from the study because they received additional treatments such as additional ESD $(\mathrm{N}=3)$, surgery $(\mathrm{N}=3)$ and radiation therapy for the non-curative resection based on the postoperative pathologic diagnosis $(N=1)$. Two patients died of non-digestive diseases and three patients had intraoperative perforation and were also excluded from our study (Fig. 1). We compared the efficacy of BTX-A injections, TA injections and controls for esophageal stricture from the remaining 78 patients. Baseline data of the patients and treatment outcomes were summarized in Table 1. There was no significant difference between the 3 groups with respect to age, sex, hospital stay days, BMI, smoking history, drinking history, family history of esophageal tumors, location of the lesion, post-operative pathology, circumferential range, longitudinal resection length, depth of infiltration, rate of en-bloc resection, operating time for ESD and adverse events such as muscular injury and hemorrhage. 
Table 1

Baseline information of the three groups

\begin{tabular}{|c|c|c|c|c|}
\hline & $\begin{array}{l}\text { BTX-A (Group } \\
\text { A) } \\
(n=26)\end{array}$ & $\begin{array}{l}\text { TA (Group } \\
\text { B) } \\
(n=16)\end{array}$ & $\begin{array}{l}\text { Control (Group } \\
\text { C) } \\
(n=36)\end{array}$ & $\begin{array}{l}p \\
\text { value }\end{array}$ \\
\hline Sex (male, n\%) & 65.4 & 62.5 & 72.2 & 0.741 \\
\hline Age (mean $\pm S D$, year) & $65.15 \pm 7.23$ & $65.06 \pm 7.88$ & $65.28 \pm 8.11$ & 0.995 \\
\hline $\begin{array}{l}\text { Hospital stay (medium } \pm \text { Interquartile } \\
\text { Range, day) }\end{array}$ & $7 \pm 2.3$ & $7 \pm 3$ & $8 \pm 4$ & 0.432 \\
\hline $\mathrm{BMI}($ mean $\pm \mathrm{SD})$ & $23.35 \pm 2.72$ & $23.47 \pm 3.04$ & $23.83 \pm 3.03$ & \\
\hline Smoking history (n\%) & 42.3 & 37.5 & 30.6 & 0.600 \\
\hline Drinking history (n\%) & 42.3 & 37.5 & 22.2 & 0.215 \\
\hline $\begin{array}{l}\text { Family history of esophageal Tumors } \\
(\mathrm{n} \%)\end{array}$ & 15.4 & 6.3 & 16.7 & 0.701 \\
\hline \multicolumn{5}{|l|}{ Location of lesion } \\
\hline Upper & 2 & 1 & 1 & 0.553 \\
\hline Middle & 13 & 10 & 25 & \\
\hline Lower & 11 & 5 & 10 & \\
\hline \multicolumn{5}{|l|}{ Post-operative pathology } \\
\hline HGIN/Carcinoma in situ & 23 & 11 & 33 & 0.097 \\
\hline Squamous cell carcinoma & 3 & 5 & 3 & \\
\hline Circumferential range & & & & 0.945 \\
\hline Two thirds to three fourths & 9 & 4 & 9 & \\
\hline Three fourths to entire & 11 & 8 & 17 & \\
\hline Full circumference & 6 & 4 & 10 & \\
\hline $\begin{array}{l}\text { Longitudinal resection length, mean } \pm S D \text {, } \\
\mathrm{cm}\end{array}$ & $4.83 \pm 1.32$ & $4.75 \pm 1.05$ & $4.69 \pm 2.00$ & 0.943 \\
\hline Depth of infiltration & & & & 0.129 \\
\hline Mucosal & 23 & 11 & 24 & \\
\hline Submucosal & 3 & 5 & 12 & \\
\hline ESD procedure time, min, median (range) & $\begin{array}{l}102.86 \pm \\
54.28\end{array}$ & $\begin{array}{l}120.00 \pm \\
69.69\end{array}$ & $\begin{array}{l}112.36 \pm \\
53.00\end{array}$ & 0.627 \\
\hline
\end{tabular}




\begin{tabular}{|c|c|c|c|c|}
\hline & $\begin{array}{l}\text { BTX-A (Group } \\
\text { A) } \\
(n=26)\end{array}$ & $\begin{array}{l}\text { TA (Group } \\
\text { B) } \\
(n=16)\end{array}$ & $\begin{array}{l}\text { Control (Group } \\
\text { C) } \\
(n=36)\end{array}$ & $\begin{array}{l}p \\
\text { value }\end{array}$ \\
\hline Rate of en-bloc resection & $24 / 26$ & $16 / 16$ & $34 / 36$ & 0.816 \\
\hline \multicolumn{5}{|l|}{ Adverse events } \\
\hline Muscular injury & 4 & 6 & 14 & 0.114 \\
\hline Hemorrhage & 1 & 1 & 4 & 0.658 \\
\hline
\end{tabular}

\section{Primary outcome}

As shown in Table 2, we found that the proportion of patients developing stricture in BTX-A group was $30.00 \%$ (intention to treat analysis, $9 / 30$ ) and $26.92 \%$ (per protocol analysis, $7 / 26$ ), in TA group was $40.90 \%$ (intention to treat analysis, $9 / 22$ ) and $43.75 \%$ (per protocol analysis, $7 / 16$ ), and in control group was $84.21 \%$ (intention to treat analysis, $32 / 38$ ) and $83.33 \%$ (per protocol analysis, $30 / 36)(p<0.001)$. When further comparing between each of the two groups, the incidence of esophageal stricture was lower in BTX-A group than that in control group $(p<0.001, \chi 2=19.964, O R=0.074(0.021-0.253))$, and lower in TA group than that in control group $(p=0.004, \chi 2=8.456, O R=0.156(0.042-0.583))$. However, the incidence of esophageal stricture was found no significantly difference between BTX-A and TA group ( $p=$ $0.261, \chi 2=1.262, \mathrm{OR}=2.111(0.567-7.855))($ Table 3$)$.

Table 2. Presentation of post-ESD esophageal stricture formation rates (per protocol set and intention to treat)

Main Outcome Measurement: The incidence of esophageal strictures

\begin{tabular}{|lllll|}
\hline & BTX-A (group & TA (group B) & Control (group & $p$ value \\
& A) & $(n=16)$ & C) & \\
& $(\mathrm{n}=26)$ & & $(\mathrm{n}=36)$ & \\
Proportion of patients developing & $30.00 \%(9 / 30)$ & $40.90 \%$ & $84.21 \%$ & $<0.001^{*}$ \\
stricture & & $(9 / 22)$ & $(32 / 38)$ & \\
(intention to treat) & & & & \\
$\begin{array}{l}\text { Proportion of patients developing } \\
\text { stricture }\end{array}$ & $26.92 \%(7 / 26)$ & $43.75 \%$ & $83.33 \%$ & $<0.001^{*}$ \\
(per protocol) & & $(7 / 16)$ & $(30 / 36)$ & \\
\hline
\end{tabular}

${ }^{*} p<0.05$ 
Table 3

Comparison of esophageal stricture incidence between each of the two groups

\begin{tabular}{|llll|}
\hline & BTX-A vs TA & BTX-A vs control & TA vs control \\
\hline $\mathrm{p}$ & 0.261 & $<0.001^{*}$ & $0.004^{*}$ \\
\hline $\mathrm{X} 2$ & 1.262 & 19.964 & 8.456 \\
\hline $\mathrm{OR}(95 \% \mathrm{Cl})$ & $2.111(0.567-7.855)$ & $0.074(0.021-0.253)$ & $0.156(0.042-0.583)$ \\
\hline${ }^{*} \mathrm{p}<0.05$ & & & \\
\hline
\end{tabular}

Subgroup analysis according to the extent of the defect after ESD was further conducted to compare the esophageal stricture rates among the three groups (Table 4). No patient in BTX-A and TA groups in the two thirds to three fourths circumference mucosal defect subgroup developed esophageal stricture. Five patients developed stricture in the two thirds to three fourths circumference mucosal defect subgroup in the control group. In the three fourths to nearly full circumference mucosal defect subgroup, $45.5 \%(5 / 11)$ patients from group A, $37.5 \%$ (3/8) patients from group B and $88.2 \%(15 / 17)$ of group C patients developed strictures. In patients with entire circumference mucosal defect subgroup, 33.3\% (3/6) of group A, 100\% (4/4) of group B and $60 \%$ (10/10) of group C patients developed strictures. Patients with full circumferential mucosal defects received additional oral prednisolone administration. Although the results of esophageal stricture rate showed that no significant difference was found in BTX-A and TA group, in entire circumference mucosal defect subgroup, the esophageal stricture was significantly lower in BTX-A group than that in TA group (33.3\% vs $100 \%, p=0.0454)$.

Table 4

Numbers of patients experiencing esophageal stricture in the three groups

\begin{tabular}{|lllll|}
\hline & $\begin{array}{l}\text { BTX-A (group A) } \\
(\mathbf{n = 2 6 )}\end{array}$ & $\begin{array}{l}\text { TA (group B) } \\
(\mathbf{n = 1 6 )}\end{array}$ & $\begin{array}{l}\text { Control (Group C) } \\
(\mathbf{n = 3 6 )}\end{array}$ & $\begin{array}{l}\text { Total stricture } \\
\text { number }\end{array}$ \\
\hline Two thirds to three fourths & $0 / 9$ & $0 / 4$ & $5 / 9$ & 5 \\
\hline Three fourths to entire & $5 / 11$ & $3 / 8$ & $15 / 17$ & 23 \\
\hline Full circumference & $2 / 6$ & $4 / 4$ & $10 / 10$ & 16 \\
\hline Total & $7 / 26$ & $7 / 16$ & $30 / 36$ & 44 \\
\hline${ }^{*}<0.05$ & & & & \\
\hline
\end{tabular}

Secondary outcome

After ESD procedures, patients in group A required an average of 1.19 bougie dilations (range, $0-12$ ), patients in group $B$ required an average of 1.31 bougie dilations (range, $0-9$ ), whereas patients in group $C$ required an average of 3.14 bougie dilations (range, $0-16)(p=0.019)$. The mean Quality of Life Questionnaire (EORTC QLQ-OES18) score in group A was $24.15 \pm 2.19$, in group B was $24.88 \pm 2.13$ and in group $C$ was $23.47 \pm 1.1(p=0.029)$. The grading of dysphagia was $0.5(0-3), 1.5(0-3)$ and $2(0-4)$ in 
group $A, B$ and $C$, respectively $(p=0.009)$. As shown in Table 5 , there was a significant difference among the three groups in the Atkinson rating of dysphagia, the Quality of Life Questionnaire (EORTCQLQOES18) scores, and the number of bougies needed after ESD $(P<0.05)($ Table 5$)$. Two representative case in the group $A$ and $B$ are shown in Figs. 2 and 3.

Table 5

Characteristics of the three groups after ESD procedure

\begin{tabular}{|c|c|c|c|c|}
\hline & $\begin{array}{l}\text { BTX-A (group } \\
\text { A) } \\
(n=26)\end{array}$ & $\begin{array}{l}\text { TA (group } \\
\text { B) } \\
(n=16)\end{array}$ & $\begin{array}{l}\text { Control (Group } \\
\text { C) } \\
(n=36)\end{array}$ & $\begin{array}{l}P \\
\text { value }\end{array}$ \\
\hline Grading of dysphagia (range) & $0.5(0-3)$ & $1.5(0-3)$ & $2(0-4)$ & $0.009^{*}$ \\
\hline $\begin{array}{l}\text { Scores of EORTC QLQ-OES18, mean } \pm \\
\text { SD }\end{array}$ & $24.15 \pm 2.19$ & $\begin{array}{l}24.88 \pm \\
2.13\end{array}$ & $23.47 \pm 1.13$ & $0.029^{*}$ \\
\hline $\begin{array}{l}\text { No. of required bougie dilations, mean } \\
\text { (range) }\end{array}$ & $1.19(0-12)$ & $1.31(0-9)$ & $3.14(0-16)$ & $0.019^{*}$ \\
\hline $\begin{array}{l}\text { Time that stricture occurred, mean } \pm S D \text {, } \\
\text { days }\end{array}$ & $43.5 \pm 37$ & $40 \pm 20$ & $32 \pm 50.5$ & 0.643 \\
\hline${ }^{*} p<0.05$ & & & & \\
\hline
\end{tabular}

Logistic regression analysis was conducted. The following factors of sex, age, smoking and drinking history, family history of esophageal tumor, location of lesion, longitudinal resection length, postoperation pathology and oral prednisolone were not relevant to the development of postoperative esophageal strictures. However, circumferential range (OR: 38.600; $95 \% \mathrm{Cl}: 2.687-554.545, \mathrm{P}=0.007$ ), BTX-A injection (OR: $0.000 ; 95 \% \mathrm{Cl}, 0.000-0.078, \mathrm{P}=0.004$ ), TA injection (OR: $0.003 ; 95 \% \mathrm{Cl}$ : 0.000-0.452, P $=0.023$ ) and depth of infiltration (OR: $0.002 ; 95 \% \mathrm{Cl}: 0.000-0.359, \mathrm{P}=0.020$ ) were shown to be risk factors for the formation of esophageal strictures after ESD. As shown in Table 6, according to the OR value, BTX-A injection, TA injection, circumferential range and depth of infiltration were protective factors for the formation of esophageal strictures after ESD. 
Table 6

Multivariate logistic regression analysis of the confounding factors in the development of postoperative esophageal strictures

\begin{tabular}{|llll|}
\hline Parameters & P value & Odds ratio & $95 \%$ Confidence interval \\
\hline Family history of esophageal tumor & 0.537 & 2.200 & $0.179-26.968$ \\
\hline Smoking history & 0.654 & 0.605 & $0.067-5.437$ \\
\hline Drinking history & 0.597 & 1.873 & $0.183-19.162$ \\
\hline ESD duration & 0.277 & 1.013 & $0.990-1.037$ \\
\hline Tumor location & 0.197 & 0.302 & $0.049-1.862$ \\
\hline post-operation pathology & 0.638 & 3.746 & $0.015-917.086$ \\
\hline Oral prednisolone & 0.913 & 1.219 & $0.035-41.966$ \\
\hline BTX-A injection & $0.008^{*}$ & 0.000 & $0.000-0.120$ \\
\hline TA injection & $0.028^{*}$ & 0.002 & $0.000-0.514$ \\
\hline Circumferential range & $0.008^{*}$ & 25.192 & $2.289-227.263$ \\
\hline Longitudinal resection length & 0.693 & 0.847 & $0.371-1.933$ \\
\hline Depth of infiltration & $0.029^{*}$ & 0.001 & $0.000-0.507$ \\
\hline *p<0.05 & & & \\
\hline
\end{tabular}

\section{Discussion}

As the increasing numbers of ESD procedures performed, treatment of esophageal stricture is of great clinical importance to improve the quality of patients' life and decrease the burden of society [13]. Previous studies have suggested that endoscopic injection of BTX-A or TA can prevent the occurrence of esophageal stenosis after ESD, when comparing with blank controls [10,11]. Our study is the first prospective clinical trial to confirm that compare with blank control, the endoscopic injection of BTX-A or TA significantly reduces the incidence of esophageal strictures after ESD treatment for early esophageal cancer. BTX-A or TA injection can also decrease the numbers of endoscopic bougie procedures required for stricture treatment. Additionally, in patients with entire circumference mucosal defect, the esophageal stricture rate was significantly lower in BTX-A injection than that in TA injection, which showed that BTX-A injection is superior to TA injection in larger circumference mucosal defect. Logistic regression analysis showed that BTX-A injection, TA injection and depth of infiltration were protective factors for the formation of esophageal strictures after ESD.

Steroids are commonly used for autoimmune and inflammatory diseases in the clinics [14]. A metaanalysis indicated that long-term oral steroid appears to be the optimal prevention method for 
postoperative stricture formation compared with single-dose steroid injection, multiple-dose steroid injection, and steroid injection combined with oral steroid [15]. Okamoto et al suggested that, endoscopic TA injection is not sufficient for preventing esophageal stricture in patients bearing mucosal defects covering more than seven-eighths of the esophageal circumference after ESD [16]. According to our study, we found that single local TA injection or combined with oral prednisolone was not enough as prophylaxis for post-ESD strictures, especially for patients with complete circumference mucosal defect. Moreover, multiple-dose TA injection with repeated endoscopic procedures demands perfect operation and may increase the risk of perforation and hemorrhage [17]. Besides, long treatment duration of oral steroid and its systemic effect may cause infection, worsen the condition of diabetes mellitus and increase the risk of osteoporosis [18].

It has been reported that the local application of BTX-A inhibited collagen deposition and fibrous connective tissue formation during the injury repair process in the skin, urethra, and joints [19]. Also, BTXA has been reported to reduce the movement of local muscles, decrease skin extension caused by muscle contraction, and limit the extent of inflammatory injury and tensile forces important in the process of scar formation [20]. Numerous studies have suggested that intralesional BTX-A injections are useful for the treatment of keloids and hypertrophic scars [21,22]. Wen et al perform a randomized case-controlled trial and showed that single-dose BTX-A injection is highly effective in preventing esophageal strictures postESD, which was the first to apply the benefits of BTX-A injections on scar formation from the field of plastic surgery to the digestive system [11].

In this study, BTX-A was injected at the level of the muscularis propria, whereas TA was injected at the base of the artificial ulcer and must be injected into the submucosal layer. A potential problem with endoscopic TA injection is the risk of delayed perforation, which may occur if the steroid is injected into the true muscular layer [23]. In order to achieve an effective administration of the steroid into the submucosal layer, operators were required to strip the lesions at the middle level of the submucosal layer to create enough space for the injection, which undoubtedly increased the difficulty of ESD procedure. Additionally, during the injection procedure, we found that the incidence of drug leakage was higher in TA injection than in BTX-A injection, which reduced the utilization ratio of TA [24].

There were several limitations of this study. First, the sample size of the study was small, and future randomized, double-blinded studies with larger sample sizes are needed. Second, the study was a singlecenter analysis and possible bias could not be eliminated.

\section{Conclusion}

Endoscopic injection of BTX-A and TA was effective and safety in preventing post-ESD esophageal strictures and can decrease the times of bougie dilation. Particularly, BTX-A injection was more effective in patients with entire circumference mucosal defect, which is of great clinical importance to esophageal stricture patients after ESD procedures. 


\section{Abbreviations}

ESD

Endoscopic Submucosal Dissection

BTX-A

botulinum toxin type $A$

TA

triamcinolone acetonide

EMR

endoscopic mucosal resection

\section{Declarations}

\section{Ethics approval and consent to participate}

The research approved by the Ethics Committee of the First Affiliated Hospital, Nanjing Medical University and informed consent was obtained from all participants included in the study, in agreement with institutional guidelines.

\section{Consent for publication}

Not Applicable

\section{Availability of data and materials}

All data generated or analyzed during the study are included in this published article.

\section{Competing interests}

The authors declare no competing interest for this article.

\section{Funding}

This study was granted by the Comprehensive Prevention and Treatment of Early Gastric Cancer (CXTDA2017033); the National Natural Science Foundation of China (81770561); the Construction Program of Jiangsu Provincial Clinical Research Center Support System (BL2014084); and the project named "The effectiveness and mechanism of local injection of botulinum toxin A for esophageal stricture after endoscopic submucosal dissection" (2017CX020).

\section{Authors' contributions}

$\mathrm{XYZ}, \mathrm{HC}$ and MHC extracted and analyzed the data; XMS planned and organized the paper; CD was responsible for follow-up and data collection; $\mathrm{HC}$ and $\mathrm{CD}$ contributed materials/analysis tools; $\mathrm{XYZ}$ and $\mathrm{MHC}$ contributed to the writing of the manuscript; GXZ and XMS proofread the paper. 
Acknowledgements

The authors thank all the patients enrolled in this study and Dr Zhirui Zhou from Fudan University Shanghai Cancer Center in providing professional statistical analysis instruction.

\section{Authors' information}

${ }^{1}$ Department of Gastroenterology, Jiangsu Province Hospital and The First Affiliated Hospital of Nanjing Medical University, Nanjing, Jiangsu, China.

\section{References}

1. Yang D, Othman M, Draganov PV. Endoscopic Mucosal Resection vs Endoscopic Submucosal Dissection For Barrett's Esophagus and Colorectal Neoplasia. Clin Gastroenterol Hepatol. 2019;17(6):1019-28.

2. Guo HM, Zhang XQ, Chen M, Huang SL, Zou XP. Endoscopic submucosal dissection vs endoscopic mucosal resection for superficial esophageal cancer. World J Gastroenterol. 2014;20(18):5540-7.

3. Sharma P, Shaheen NJ, Katzka D, Bergman JJGHM. AGA Clinical Practice Update on Endoscopic Treatment of Barrett's Esophagus With Dysplasia and/or Early Cancer: Expert Review. Gastroenterology. 2020;158(3):760-9.

4. Yamamoto Y, Kikuchi D, Nagami Y, et al. Management of adverse events related to endoscopic resection of upper gastrointestinal neoplasms: Review of the literature and recommendations from experts. Dig Endosc. 2019;31(S1):4-20.

5. Probst A, Aust D, Märkl B, Anthuber M, Messmann H. Early esophageal cancer in Europe: endoscopic treatment by endoscopic submucosal dissection. Endoscopy. 2015;47(2):113-21.

6. Siersema PD. Treatment options for esophageal strictures. Nature Clinical Practice Gastroenterology Hepatology. 2008;5:142-52.

7. Lian JJ, Ma LL, Hu JW, et al. Endoscopic balloon dilatation for benign esophageal stricture after endoscopic submucosal dissection for early esophageal neoplasms. J Dig Dis. 2014;15(5):224-9.

8. Yu JP, Liu YJ, Tao YL, et al. Prevention of Esophageal Stricture After Endoscopic Submucosal Dissection: A Systematic Review. World J Surg. 2015;39(12):2955-64.

9. Qiu Y, Shi R. Roles of Steroids in Preventing Esophageal Stricture after Endoscopic Resection. Can J Gastroenterol Hepatol. 2019; 2019: 5380815.

10. Chu Y, Chen T, Li H, et al. Long-term efficacy and safety of intralesional steroid injection plus oral steroid administration in preventing stricture after endoscopic submucosal dissection for esophageal epithelial neoplasms. Surg Endosc. 2019;33(4):1244-51.

11. Wen J, Lu Z, Linghu E, et al. Prevention of esophageal strictures after endoscopic submucosal dissection with the injection of botulinum toxin type A. Gastrointest Endosc. 2016;84(4):606-13. 
12. Xiao Z, Zhang M, Liu Y, Ren L. Botulinum toxin type a inhibits connective tissue growth factor expression in fibroblasts derived from hypertrophic scar. Aesthetic Plast Surg. 2011;35(5):802-7.

13. Kawaguchi K, Kurumi H, Takeda Y, Yashima K, Isomoto H. Management of strictures after endoscopic submucosal dissection for superficial esophageal cancer. Ann Transl Med. 2017;5(8):184.

14. Straub RH, Cutolo M. Glucocorticoids and chronic inflammation. Rheumatology. 2016;55(suppl 2):ii6-14.

15. Yang J, Wang X, Li Y, et al. Efficacy and safety of steroid in the prevention of esophageal stricture after endoscopic submucosal dissection: A network meta-analysis. J Gastroenterol Hepatol. 2019;34(6):985-95.

16. Okamoto K, Matsui S, Watanabe T, et al. Clinical Analysis of Esophageal Stricture in Patients Treated with Intralesional Triamcinolone Injection after Endoscopic Submucosal Dissection for Superficial Esophageal Cancer. Oncology. 2017;93(Suppl 1):9-14.

17. Yamamoto Y, Kikuchi D, Nagami Y, et al. Management of adverse events related to endoscopic resection of upper gastrointestinal neoplasms: Review of the literature and recommendations from experts. Dig Endosc. 2019;31(Suppl 1):4-20.

18. lizuka T, Kikuchi D, Hoteya S, Kaise M. Effectiveness of modified oral steroid administration for preventing esophageal stricture after entire circumferential endoscopic submucosal dissection. Dis Esophagus. 2018;31(7):10.

19. Namazi H, Torabi S. Novel use of botulinum toxin to ameliorate arthrofibrosis: an experimental study in rabbits. Toxicol Pathol. 2007;35:715-8.

20. Sahinkanat T, Ozkan KU, Ciralik H, et al. Botulinum toxin-A to improve urethral wound healing: an experimental study in a rat model. Urology. 2009;73:405-9.

21. Zhibo X, Miaobo Z. Intralesional botulinum toxin type A injection as a new treatment measure for keloids. Plast Reconstr Surg. 2009;124:275-7.

22. Kasyanju Carrero LM, Ma WW, Liu HF, Yin XF, Zhou BR. Botulinum toxin type A for the treatment and prevention of hypertrophic scars and keloids: Updated review. J Cosmet Dermatol. 2019;18(1):10-5.

23. Kochhar R, Makharia GK. Usefulness of intralesional triamcinolone in treatment of benign esophageal strictures. Gastrointest Endosc. 2002;56:829-34.

24. Ishida T, Morita $\mathrm{Y}$, Hoshi N, et al. Disseminated nocardiosis during systemic steroid therapy for the prevention of esophageal stricture after endoscopic submucosal dissection. Digestive Endoscopy. 2015;27:388-91.

\section{Figures}




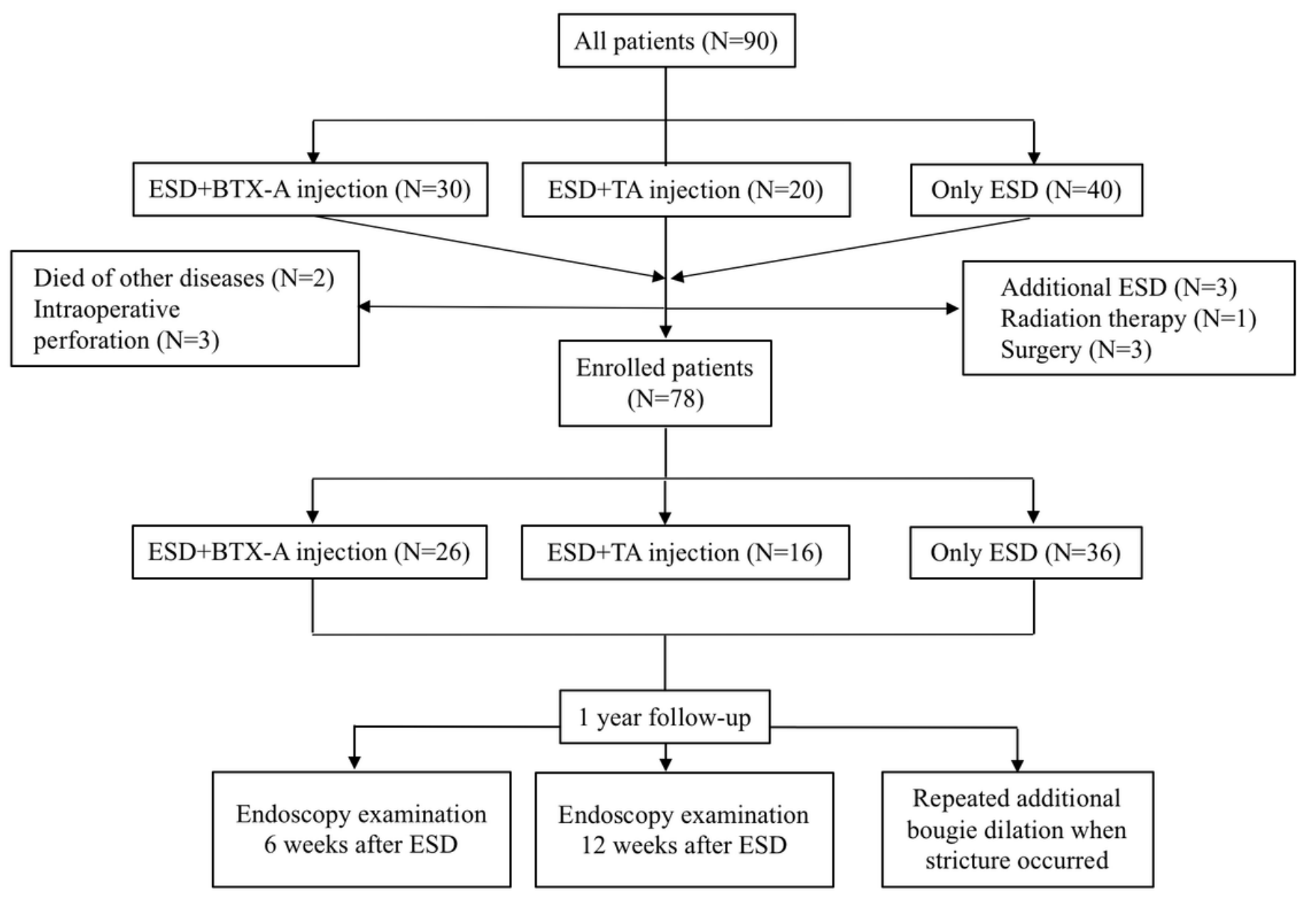

\section{Figure 1}

Flow diagram showing inclusion and exclusion of patients for the three study groups. ESD, endoscopic submucosal dissection; BTX-A, botulinum toxin type A; TA, triamcinolone acetonide.
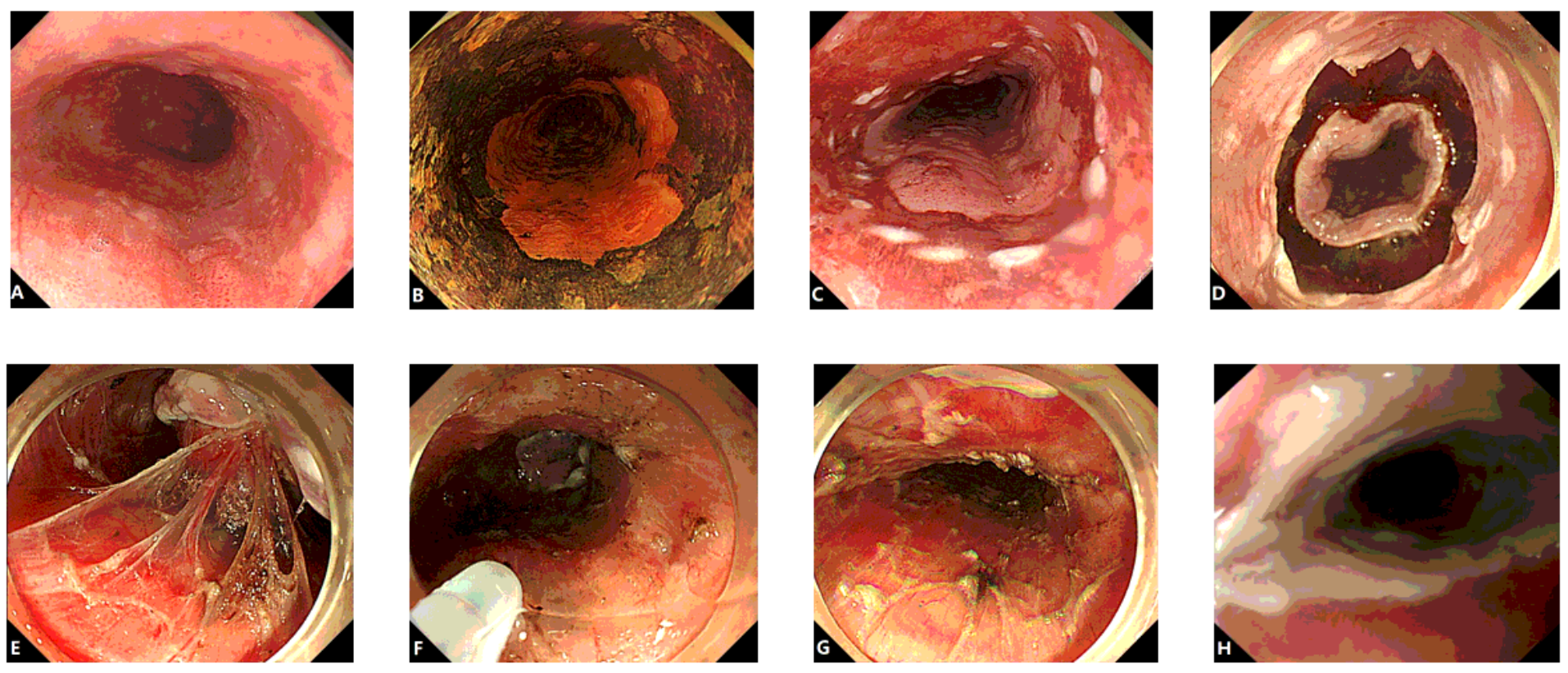


\section{Figure 2}

ESD procedures and postoperative gastroscope in patients receiving BTX-A injection. (A) Early esophageal cancer extending entire circumferential of the esophageal lumen. (B) Lugol's iodine solution was administered, and the lesion area was not stained. (C) Lesion was marked and the lift sign was positive. (D-E) The edge of the lesion was cut and separated step by step. (F) BTX-A was injected at muscularis propria by points. (G) The artificial ulcer caused by ESD, which resulted in a mucosal defect affecting entire circumference. $(\mathrm{H})$ Follow-up endoscopy 6 weeks after ESD treatment revealed a white scar and no postoperative stricture.
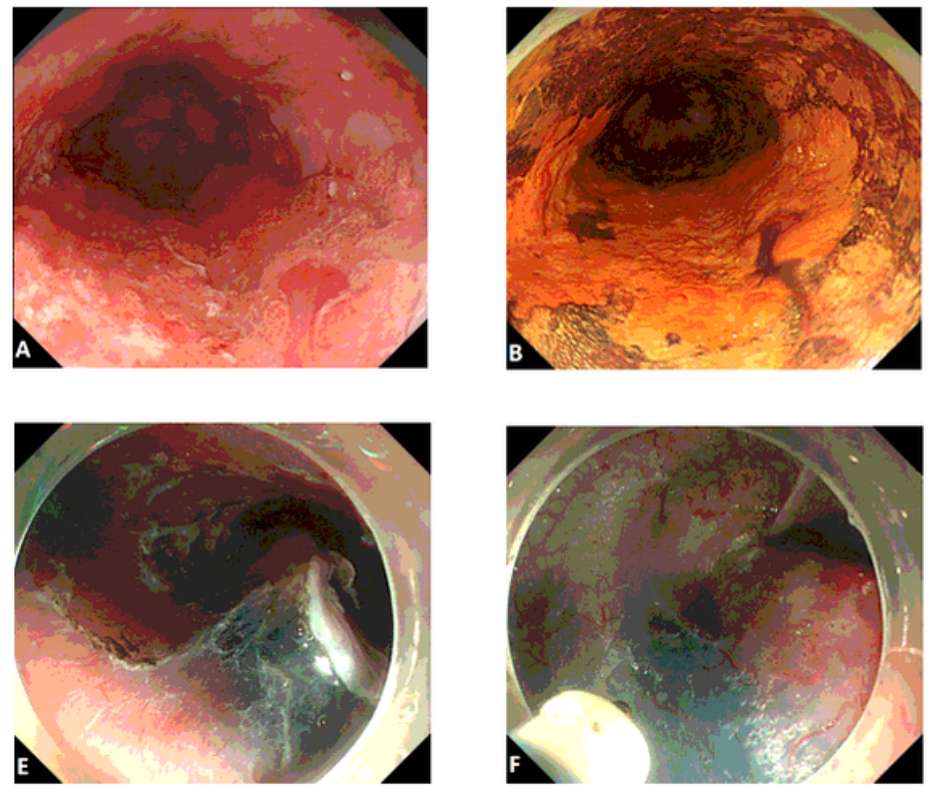
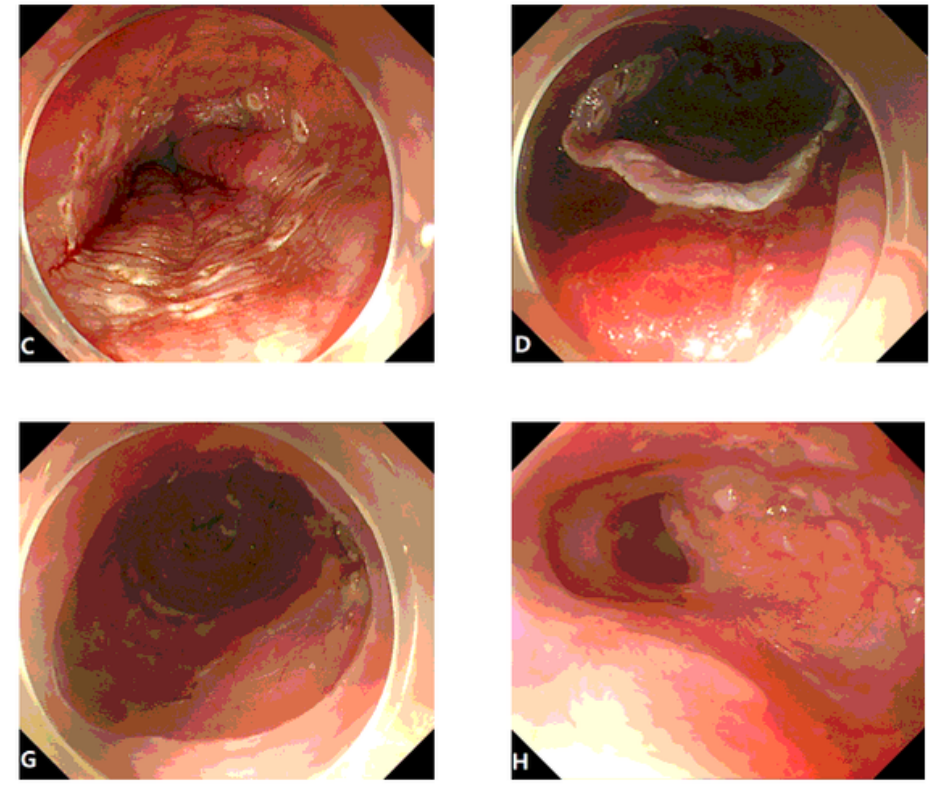

Figure 3

ESD procedures and postoperative gastroscope in patients receiving TA injection. (A) Early esophageal cancer extending nearly four fifths of the circumference of the esophageal lumen. (B) Lugol's iodine solution was administered, and the lesion area was not stained. (C) Lesion was marked and the lift sign was positive. (D-E) The edge of the lesion was cut and separated step by step. (F) The artificial ulcer caused by ESD, which resulted in a mucosal defect affecting more than four fifths of the circumference. (H) Follow-up endoscopy 6 weeks after ESD treatment revealed a white scar and no postoperative stricture. 